\title{
Force generation in small ensembles of Brownian motors
}

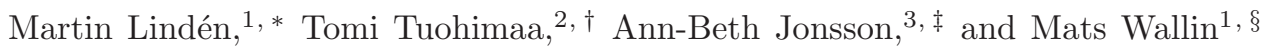 \\ ${ }^{1}$ Theoretical Physics, Royal Institute of Technology, AlbaNova, 10691 Stockholm, Sweden \\ ${ }^{2}$ Applied Physics, Royal Institute of Technology, AlbaNova, 10691 Stockholm, Sweden \\ ${ }^{3}$ Department of Medical Biochemistry and Microbiology, \\ Uppsala Biomedical Center, Uppsala University, Box 582, 75123 Uppsala, Sweden
}

(Dated: 12th November 2018)

\begin{abstract}
The motility of certain gram-negative bacteria is mediated by retraction of type IV pili surface filaments, which are essential for infectivity. The retraction is powered by a strong molecular motor protein, PilT, producing very high forces that can exceed $150 \mathrm{pN}$. The molecular details of the motor mechanism are still largely unknown, while other features have been identified, such as the ring-shaped protein structure of the PilT motor. The surprisingly high forces generated by the PilT system motivate a model investigation of the generation of large forces in molecular motors. We propose a simple model, involving a small ensemble of motor subunits interacting through the deformations on a circular backbone with finite stiffness. The model describes the motor subunits in terms of diffusing particles in an asymmetric, time-dependent binding potential (flashing ratchet potential), roughly corresponding to the ATP hydrolysis cycle. We compute force-velocity relations in a subset of the parameter space and explore how the maximum force (stall force) is determined by stiffness, binding strength, ensemble size, and degree of asymmetry. We identify two qualitatively different regimes of operation depending on the relation between ensemble size and asymmetry. In the transition between these two regimes, the stall force depends nonlinearly on the number of motor subunits. Compared to its constituents without interactions, we find higher efficiency and qualitatively different force-velocity relations. The model captures several of the qualitative features obtained in experiments on pilus retraction forces, such as roughly constant velocity at low applied forces and insensitivity in the stall force to changes in the ATP concentration.
\end{abstract}

PACS numbers: 87.16.-b,05.40.-a

\section{INTRODUCTION}

Recent experimental progress has enabled remarkable quantitative measurement of biological processes on the single-molecule level [1]. One example is the biomechanics of force generation by molecular machines such as kinesin, myosin, and dynein [2, 3]. This has stimulated considerable modeling activity in order to analyze the experiments $[2,3,44,5,6]$. In this paper we are inspired by another motor protein, called PilT [], which has interesting properties; e.g., it is the strongest known molecular motor [8].

The PilT motor is responsible for the retraction of certain bacterial surface filaments, and the velocities and forces generated during retraction have been measured in a series of laser tweezers experiments [8, 9, 10]. Theoretical analysis of the retraction data has revealed interesting information about the underlying retraction mechanism [10]. It is also of interest to study the question of how large the generated forces can be, given the energy and length scales relevant to the PilT motor, and what features are important for generation of large forces. We will address this question through a simple ratchet model,

\footnotetext{
*Electronic address: linden@kth.se

${ }^{\dagger}$ Electronic address: tomi.tuohimaa@biox.kth.se

¥Electronic address: Ann-Beth.Jonsson@imbim.uu.se

$\S$ Electronic address: wallin@kth.se
}

which is inspired by known experimental facts of the PilT system.

Ratchet models of particles in fluctuating potentials are commonly used in theoretical studies of molecular motors [4, 11. Single-particle models have been used to study kinetics of ATP consumption in molecular motors 12. and to describe the kinetics of kinesin 13. Models of particles in ratchet potentials have also been employed to describe collective effects in large ensembles of interacting motors 14, 15, 16, 17, 18, 19, 20. Finite ensembles of Brownian particles have been studied to some extent in the context of two-headed motor proteins 21, 22, 23, 24, 25] and to describe the bacterial flagella motor [26].

Another approach, in which the motion of a molecular motor is described in terms of transitions between discrete chemical and conformational states, has also been generalized to the case of two interacting motor subunits 27. As is evident from Refs. 14, 15, 16, 17, 18, 19, 20, 21, 22, 23, 24, 25, 26], interactions among several motors can lead to new and nontrivial behavior of the average velocity, which is not present if the interaction is turned off. It is natural to ask if this is the case for force generation as well.

In this paper, we investigate the behavior of a small ensemble of interacting processive Brownian motors and focus on the effect of the interaction on the generation of large forces. We aim at a prototypical, minimal model which captures certain features of the PilT system. The main input is the overall structures of the filament and PilT complex. When possible, we also use the exper- 
imental situation to estimate model parameters, which should ideally be as few as possible. The model is a generalization of the model of two elastically coupled motors studied by Dan et al. 24] to larger ensembles, but focuses on different properties. By varying the density of motor subunits and other parameters of the system, we explore the force production in different regimes. We compare with a single building block of our model to identify the effect of the interactions and also compare with experimental results. Although the detailed connection between the model and the actual molecular retraction mechanism is speculative, the spirit of the model is best understood in light of the known facts about the PilT system. Therefore, we will briefly review some facts about pilus retraction before introducing the model.

Type IV pili are surface filaments crucial for the initial adherence of certain gram-negative bacteria to target host cells, DNA uptake, cell signaling, and bacterial motility [7]. Each filament consists of thousands of pilin monomers that polymerize to a helical structure with outer diameter of about $6 \mathrm{~nm}, 4 \mathrm{~nm}$ pitch, and five monomers per turn 7,28 . The bacterial motility associated with type IV pili, called twitching motility, is driven by repeated extension, tip attachment, and retraction of the pilus filament, by which the bacterium can pull itself forward on surfaces like glass plates or target host cells [9]. Type IV pili are expressed by a wide range of gram-negative bacteria [7] including Myxococcus xanthus 29] and human pathogens Neisseria gonorrhoeae [9] and Pseudomonas aeruginosa [30].

The mechanism of retraction is believed to be filament disassembly mediated by PilT, a member of the AAA family of motor proteins [7], but the microscopic details of this process are not known. One might compare pilus retraction with force generation by microtubules, which are multistranded filaments with effective monomer lengths similar to type IV pili. The helical pitch divided by the number of filament strands is $0.8 \mathrm{~nm}$ for the pili and $0.6 \mathrm{~nm}$ for microtubules 3 . However, pilus retraction generates forces of up to $160 \mathrm{pN}$ [8, 10], which is an order of magnitude larger than those observed in in vitro experiments on microtubules [3, 31, 32]. Another difference is that the pilus retraction velocity is independent of filament length [8]. Since dissociated pilin monomers are stored in the cell membrane waiting to be recycled in other filaments [30], the implication is that the velocity is independent of pilin concentration in the membrane. This presumably rules out simple polymerization ratchet-type models, which have been proposed to describe polymerization forces generated by microtubules [3, 31]. The experimental evidence instead favors a retraction process driven by an active molecular motor [8]

Pilus retraction is highly processive, and retraction velocities are of the order of $0.5-1 \mu \mathrm{m} / \mathrm{s}$ [7, 8, , 10, 30]. Generation of high forces persists when the PilT concentration is reduced, suggesting that one single PilT complex retracts the pilus filament [8]. The stall force (the force at which the average velocity drops to zero) and the veloc- ity at high forces are insensitive to changes in ATP concentration, and the retraction velocity is roughly forceindependent for small applied forces $(\lesssim 50 \mathrm{pN})$ within the experimental accuracy [8, 10]. PilT has been shown to form a ring structure with sixfold symmetry [33], and since each subunit has an ATP binding motif, it is possible that it can hydrolyze up to six ATP molecules in parallel during retraction [7]. The outer diameter of the ring is about $10 \mathrm{~nm}$, and the inner diameter varies in the range $2-4 \mathrm{~nm}$ [33.

Pilus retraction is interesting from a technological point of view, as a potential prototype for a nanomachine that can generate large forces, and from a biomedical point of view since pilus retraction is important for the infectivity of various severe bacterial pathogens 34].

There are several proposals for how the molecular constituents of the retraction machinery fit together. One of them is that PilT forms a ring around the base of the pilus [7, 35]. The hole in the middle of the PilT complex seems too small to let the assembled filament through, but large enough for pilin monomers. This could allow interactions between the pilus and PilT via several active sites (motor subunits) that work together and is the principle that we will explore here. For simplicity, we assume one motor subunit per filament strand and neglect possible two-dimensional effects such as angular motion of the filament.

We stress that the purpose of this paper is not to attempt to describe the detailed molecular mechanisms involved in pilus retraction, which are largely unknown. Rather we examine a new regime of a simple model, whose main features are inspired by experiments. Below we obtain several results from the model, such as large force generation and other properties that agree well with interesting experimental results on pilus retraction. Moreover, these results are a consequence of correlations and interactions between the motor subunits and are strikingly different from the characteristics of the single building block of the model.

\section{RETRACTION MODEL}

In this section the geometry and equations of motion of the model are described. We then discuss the parameters, which come in several kinds: parameters that are known for the PilT system, parameters that can be estimated to varying degrees of accuracy, and parameters that we will explore in a systematic way. A few parameters cannot be estimated due to the lack of knowledge of the molecular details. In this case, we make an arbitrary choice in order to investigate the qualitative behavior of the model. The basic setup is sketched in Fig. पa). A ring of $M$ motor subunits interacts with an $M$ stranded helical filament, with repeat distance $d$ of the single strands. The filament coordinate $q$ decreases during retraction. We also allow for deformations of the PilT ring. This is described by displacements $x_{i}$ of the mo- 

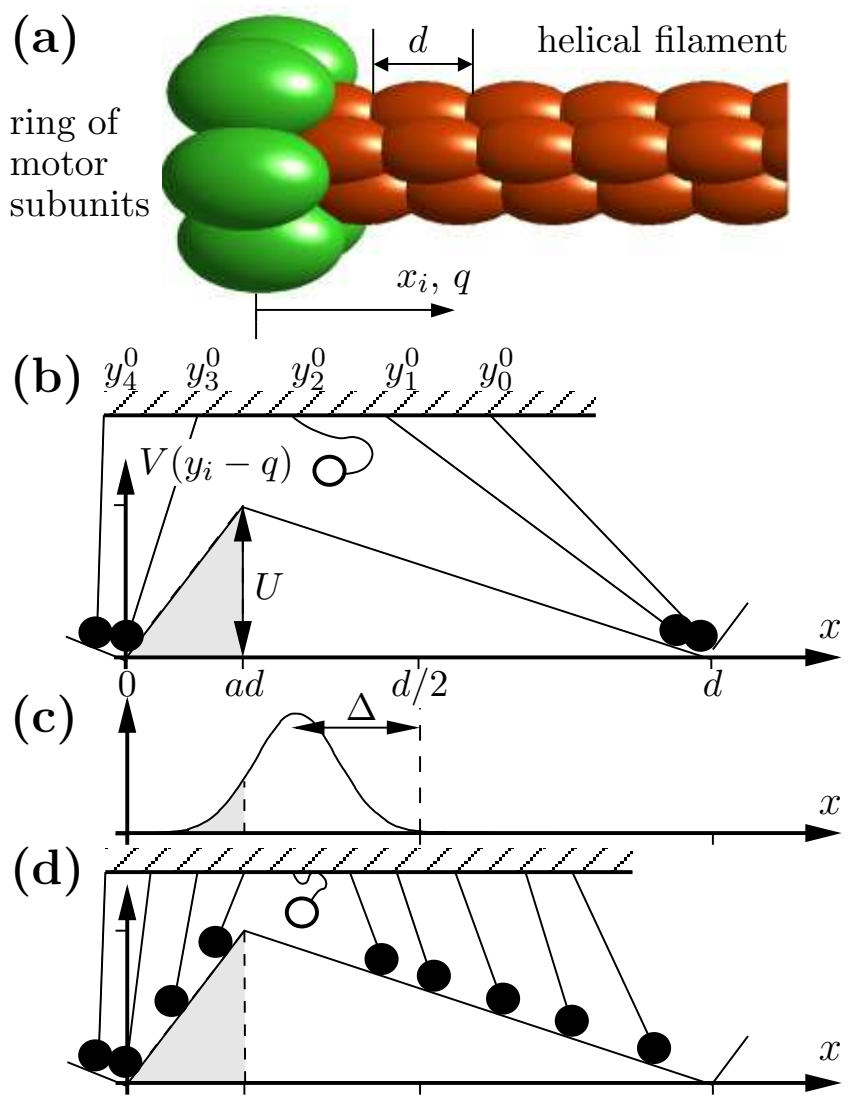

Figure 1: (Color online) (a) The elements of the retraction motor model consist of a flexible ring of motor subunits that interact with a moving helical filament. (b) Equivalent geometry after the change of variables $y_{i}=x_{i}-i d / M$, which places the binding potentials of the filament monomers on top of each other. The binding potential is assumed to be an asymmetric ratchet potential with amplitude $U$ and asymmetry $a$. The undeformed state of the motor protein complex is described by the equilibrium positions $y_{i}^{0}$ of the motor subunits, and the subunits are elastically confined to their equilibrium positions. Due to the helical structure of the filament, the equilibrium positions become evenly spread over one period. The motor subunits at positions $y_{i}$ (black circles) interact with the filament potential. The open circle represents an unbound subunit. During a successful retraction process, a motor subunit detaches from the filament, relaxes in its confinement potential, and rebinds near the next binding site along the filament. (c) Distribution of the unbound subunit in B. The shaded area represents the probability for the subunit to bind to the left of the potential maximum and produce a failed step. As the applied force increases, the filament is pulled forward relative to the distribution and the probability of a failed step increases. (d) Qualitatively new behavior emerges in the limit of high stiffness (strong confinement) and many motor subunits. In this limit, all bound motor subunits will not relax to the minima in the binding potential. Instead, some subunits will interact with the shaded part of the potential and oppose force production. In this regime, one expects that the stall force depends nonlinearly on the number of motor subunits, and nonmonotonically on the stiffness. tor subunits from the undeformed state. We assume that the motor subunits interact with the filament strands via identical one-dimensional binding potentials with period $d$. To mimic the helical structure of the filament, the potential of subunit $i$ is displaced a distance $i d / M$ relative to the potential of subunit 0 . The interactions between the filament and subunit $i$ therefore have the form $V\left(x_{i}-q-i d / M\right)$, where $V$ is some one-dimensional binding potential. We formulate the equations of motion as a system of overdamped Langevin equations for $q$ and $x_{i}$,

$$
\begin{aligned}
\alpha \dot{x}_{i} & =-k x_{i}-h_{i}(t) \frac{\partial V\left(x_{i}-q-i d / M\right)}{\partial x_{i}}+\sqrt{2 \alpha k_{\mathrm{B}} T} \xi_{i}(t), \\
\gamma \dot{q} & =\sum_{i=0}^{M-1} h_{i}(t) \frac{\partial V\left(x_{i}-q-i d / M\right)}{\partial x_{i}}+F+\sqrt{2 \gamma k_{\mathrm{B}} T} \xi_{\mathrm{q}}(t),
\end{aligned}
$$

where $i=0, \ldots, M-1, \alpha$ and $\gamma$ are friction coefficients of the motor subunits and the filament respectively, $k$ is a spring constant describing the stiffness of the PilT ring, $h_{i}(t)=0,1$ are chemical state variables, $k_{\mathrm{B}}$ is Boltzmann's constant, $T$ is the temperature, $F$ is an external force acting on the filament, and $M$ is the number of motor subunits and filament strands. Thermal fluctuations are included through independent Gaussian white noise terms $\xi_{i}(t)$ and $\xi_{\mathrm{q}}(t)$, which obey $\left\langle\xi_{i}(t)\right\rangle=\left\langle\xi_{\mathrm{q}}(t)\right\rangle=0,\left\langle\xi_{j}(t) \xi_{i}\left(t^{\prime}\right)\right\rangle=\delta_{i j} \delta\left(t-t^{\prime}\right)$, and $\left\langle\xi_{\mathrm{q}}(t) \xi_{\mathrm{q}}\left(t^{\prime}\right)\right\rangle=\delta\left(t-t^{\prime}\right)$ and have prefactors according to the fluctuation-dissipation theorem. The temperature is set to $T=310 \mathrm{~K}$.

Before discussing the terms in the equations of motion in detail, we transform the subunit coordinates to place the binding potentials on top of each other. The new coordinates are convenient in order to analyze the model and are close to previous works with similar models [23, 24, 25]. The transformed subunit coordinates are $y_{i}=$ $x_{i}-i d / M+d$. An undeformed PilT ring is now described as $y_{i}=y_{i}^{0}=d-i d / M$, which we call the equilibrium positions of the subunits. The transformed equations of motions are

$$
\begin{aligned}
\alpha \dot{y}_{i} & =-k\left(y_{i}-y_{i}^{0}\right)-h_{i}(t) \frac{\partial V\left(y_{i}-q\right)}{\partial y_{i}}+\sqrt{2 \alpha k_{\mathrm{B}} T} \xi_{i}(t) \\
\gamma \dot{q} & =\sum_{i=0}^{M-1} h_{i}(t) \frac{\partial V\left(y_{i}-q\right)}{\partial y_{i}}+F+\sqrt{2 \gamma k_{\mathrm{B}} T} \xi_{\mathrm{q}}(t)
\end{aligned}
$$

Note that the binding potentials are all on top of each other, as if all motor subunits were interacting with just one single strand. The price for this convenience is that the equilibrium positions are evenly distributed over one period of the potential. The setup in the transformed variables is sketched in Fig. 1(b).

The motor subunits can be in one of two states: unbound $\left(h_{i}=0\right)$, in which they diffuse around their respective equilibrium positions, or bound $\left(h_{i}=1\right)$, in which they also interact with the filament through the binding 
potential $V\left(y_{i}-q\right)$. We treat the number of motor subunits $M$ as a parameter, and we will present results for $M=2,3,5,6$, and 12 .

For simplicity, we model the binding of the motor to the filament by an asymmetric sawtooth potential, shown in Fig. 1(c). For the asymmetry factor we take $a=0.1$, if not stated otherwise. Some asymmetry is needed to give a preferred direction of motion, and the sawtooth potential was selected to give a simple parametrization of the (unknown) real interaction potential. One possible origin of an asymmetric binding potential is surface charges on the head of the pilin monomer [36] - for example as described in Ref. [2]. Another alternative is that the asymmetry can be viewed as an effective description of some asymmetry somewhere else in the system, e.g., in the direction of the motor steps (power strokes). Based on the helical structure of the pilus filament, we take the periodicity of the potential to be $d=4 \mathrm{~nm}$ [7, 28], which we use for all values of $M$.

The amplitude $U$ of the potential is the maximal energy required to break the bond between the filament and the active site. Pilus retraction is powered by hydrolysis of one or a few ATP per retracted pilin monomer 8], which sets the energy scale for the potential. Depending on conditions, the free energy yield from hydrolysis of one ATP in a cell is about $80-100 \mathrm{pN} \mathrm{nm} \mathrm{[3].} \mathrm{The} \mathrm{motor}$ subunits are bound together to form the motor complex, and we model their confinement with a harmonic restoring force $-k x_{i}=-k\left(y_{i}-y_{i}^{0}\right)$. This linear approximation is reasonable if the deformations $x_{i}=y_{i}-y_{i}^{0}$ are small, which will be verified below.

For the binding processes $h_{i}(t)$, we restrict ourselves to a sequential reaction scheme with $M$ chemical states. We define state $j$ as $h_{k}(t)=1-\delta_{k j}$; i.e., subunit $j$ is unbound and the other subunits are bound. The states are visited in ascending order, and the (constant) transition rate from state $j$ to $j+1(\bmod M)$ is $\lambda$. The bound subunits spend most of their time near a minimum in the binding potential, and several geometrical configurations are compatible with each chemical state. The main pathway for efficient retraction in the model is that the subunits take turns to hop forward to the next minimum as they release and rebind to the filament (successful steps). When the retraction is less efficient - for example at large applied force - the subunits sometimes do not hop forward (failed step), which leads to geometrical configurations outside the main pathway. During a successful step, a motor subunit goes through the following sequence of events:

(i) The subunit is released from a minimum in the binding potential.

(ii) The released subunit relaxes to its equilibrium position. The filament relaxes in the opposite direction due to the forces from the other motor subunits.

(iii) The subunit rebinds, close to the next minimum in the binding potential (otherwise the step fails). At the same time, the next motor subunit enters step (i).

(iv) The subunit stays bound and pulls on the filament as the other $M-1$ subunits go through steps (i)(iii). After each successful rebinding event, the filament retracts a distance $d / M$.

This mechanism relies on the asymmetry of the potential and is similar to the mechanisms studied earlier for two elastically coupled particles [24, 25]. We argue below that generation of strong forces in this model relies on a binding process that always keeps several motor subunits bound to the filament, but the binding order is less important.

We now discuss the parameters of the model. In the laser tweezers experiments 8, 9, 10], the outer filament tip binds to an external latex bead with diameter $1-2$ $\mu \mathrm{m}$. Using Stokes law, $\gamma=6 \pi \eta R$, the approximate viscosity $\eta=10^{-8} \mathrm{pN} \mathrm{s} / \mathrm{nm}^{2}$ of the bulk solution surrounding the cell (somewhere between $10^{-9}$ for water and $8 \times 10^{-7}$ for glycerin seems reasonable) and $R=1 \mu \mathrm{m}$, we get $\gamma \approx 2 \times 10^{-4} \mathrm{pN} \mathrm{s} / \mathrm{nm}$ for the bead. As a first approximation, we neglect the elasticity and friction of the pilus filament itself. For the internal friction coefficient, we use $\alpha=0.5 \times 10^{-3} \mathrm{pN} \mathrm{s} / \mathrm{nm} \ll k / \lambda$. This sets the time scale for internal relaxation $\alpha / k$ much smaller than the typical time $(M-1) \lambda^{-1}$ between binding and release of individual motor subunits and lets the motor subunits reach thermal equilibrium between transitions. This is consistent with estimates of thermal relaxation times over length scales on the order of $10 \mathrm{~nm}$ [20], which is the size of the PilT ring. Another time scale for internal relaxation is given by the time to slide down to a potential minimum, $\alpha d^{2} / U$, which we also keep smaller than $(M-1) \lambda^{-1}$. We then expect the velocity to be proportional to $\lambda$, and we will restrict ourselves to this quasistatic regime for two reasons. First, this is the biologically relevant regime where we expect the stall force to be independent of $\lambda$, which corresponds to the experimental observation that the stall force is independent of ATP concentration [8]. Second, the exact value of $\alpha$ is not critical for the results in this regime, and since it is difficult to estimate $\alpha$ accurately, we can avoid making our results depend strongly on an unknown parameter.

Having found useful values for the potential period $d$ and the drag coefficients $\alpha_{i}$ and $\gamma$, we go on to investigate the model behavior as a function of the remaining parameters $a, M, k, U$, and $\lambda$ and properties of the binding process.

\section{RESULTS}

\section{A. Methods}

Retraction of the filament means that $q$ decreases, so it is natural to study the retraction velocity $v=-d q / d t$. In the laser tweezers experiment, the tip of the bead is 
held by a static laser trap which is to good approximation a harmonic potential - i.e., $F=-k_{\mathrm{t}} q$, with $k_{\mathrm{t}}$ on the order of $0.1 \mathrm{pN} / \mathrm{nm}$. Numerical solution of Eq. (1) using a standard method, known as the Milstein scheme [37, produces a deflection trajectory similar to the experimental ones. We calculate the retraction velocity by fitting a second-order polynomial to a small time interval around a point $q=-F / k_{\mathrm{t}}$ and take the velocity $v(F)$ as the derivative of the polynomial. This is similar in spirit to how the experimental data was analyzed 8, 9, 10]. The retracted distance $-q(t)$ increases from the initial value towards a steady-state, corresponding to the maximal applied force (stall force), which we define as the mean applied force in the steady state. To check our simulation code, we reproduced analytical results for the steady state current in a flashing ratchet model [38].

\section{B. Stall force and force-velocity relation}

The stall force and force-velocity relation of the motor is determined by several competing mechanisms, which we now describe qualitatively.

In the case of a few motor subunits, stalling of the retraction is controlled by two different mechanisms. One comes from the finite binding energy between the filament and the subunits. Due to the simple shape of the potential, we can estimate an upper limit for the stall force, using force $=\Delta V / \Delta q$. The maximum force that each subunit can exert on the filament during retraction against an opposing (positive) force is $U /(1-a) d$, so $M-1$ bound motor subunits give an upper limit of $U(M-1) / d(1-a)$ for the stall force. At finite temperatures the upper limit is not reached, since the motor subunits diffuse and can pass between potential minima by thermal excitation. We think of these thermally assisted transitions as slipping events, and they occur more often when the subunits are far from their equilibrium positions and experience a large confining force. This mechanism tends to increase the stall force with increased binding strength $U$.

The other mechanism has to do with the stiffness $k$ and the probability for a step to fail. This probability depends on the distribution of the filament position relative to the unbound subunit, which is illustrated in Fig. 1(c). The shaded area represents the probability of a failed step. Higher probability of failure gives lower velocity. Increasing the applied force $F$ at constant stiffness pulls the filament to the right, increases the fraction of failed steps, and decreases the velocity. If all steps fail, no retraction takes place. The width of the position distribution of a subunit in the harmonic confinement is $\sqrt{k_{\mathrm{B}} T / k}$, but the distribution in Fig. 1(c) is broader, since the filament also fluctuates. The average relative position $\Delta$ is roughly proportional to $F / k$. At very low stiffness, the distribution is broad enough for some steps to fail without applied load, and the average relative position varies strongly with applied force. This

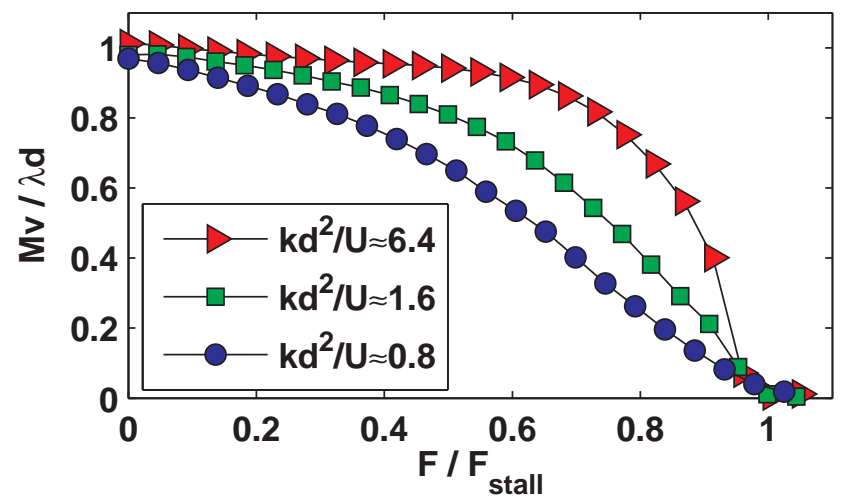

Figure 2: (Color online) Force-velocity relations for high (triangles), intermediate (squares) and low stiffness (circles) calculated for $M=5$. The y axis is velocity normalized by $\lambda d / M$, which corresponds to the velocity for $M$ motor subunits if all steps were successful.

gives a monotonically decreasing force-velocity relation and a low stall force limited by the stiffness. At very high stiffness, the distribution is narrow and the average relative position is less sensitive to the applied force. Almost no steps fail without applied load, and it takes some threshold force before steps start to fail significantly. We get a force-velocity relation that is almost independent of force at low forces, and the stall force is mainly limited by slipping events. Figure 2 shows examples of normalized force-velocity relations of the model for strong, intermediate, and weak stiffness, compared to the binding strength. The curves illustrate the qualitative arguments of the preceding paragraphs. We also find that the stall force is insensitive to changes in the reaction rate $\lambda$ (not shown). Since the reaction rate corresponds to the ATP concentration, this is in qualitative agreement with the experimental result that the stall force is insensitive to the ATP concentration [8]. This is expected in the quasistatic regime that results from the choice of time scales discussed at the end of Sec.II Stiff systems $\left(k d^{2} / U \gg 1\right)$ have a plateau in the retraction velocity at low forces, which is also the general experimental trend 8, 10]. For the parameter regime we have investigated, Fig. 2 gives the qualitative shape of the force-velocity relation as a function of $k / U$. The size of the plateau is roughly proportional to the stall force, with a proportionality constant that is to first approximation a function only of $k / U$.

As the number of motor subunits and filament strands increases, high stiffness can also have a destructive effect on the stall force, as illustrated in Fig. 1D. This occurs when the distance between equilibrium positions is shorter than the region of the binding potential with backward slope (shaded) - i.e., $M>1 / a$. In that case, some of the bound motor subunits will tend to interact with the shaded region of the potential if the stiffness is high. There they act with a negative force on the filament 
and contribute negatively to the force production. This effect is enhanced by increased stiffness, and we therefore expect the stall force to have a maximum as a function of stiffness. This is a qualitatively different behavior than with only a few motor subunits $(M<1 / a)$ and makes the stall force depend in a highly nonlinear way on $M$.

\section{Parametrization of the stall force}

Equation (2) suggests that the stall force might depend on the ratio $k / U$, instead of $k$ and $U$ independently. We will use this observation to further analyze force production in the model. A parametrization of the stall force is obtained from a combination of the estimated upper limit $U(M-1) / d(1-a)$ for the stall force with an function of $k / U$. Using this (unknown) function $f_{M}$, which also depends on $M$, we describe the effect of stiffness in the following way:

$$
F_{\text {stall }}(k, U)=F_{\text {inf }} f_{M}\left(\frac{k d^{2}}{U}\right), \quad F_{\text {inf }}=\frac{M-1}{d(1-a)}\left(U-U_{M}\right),
$$

where $U_{M}$ is a free $M$-dependent parameter, independent of $U$ and $k$, and $d^{2}$ was inserted to make the argument of $f_{M}$ dimensionless. One can obtain $f_{M}(x)$ by plotting $F_{\text {stall }} / F_{\text {inf }}$ against $k d^{2} / U$ and adjusting $U_{M}$. Such a plot is shown in Fig. 3(a), with the best fit values of $U_{M}=25.4,25.9,28.9,28.3$, and $33.5 \mathrm{pN} \mathrm{nm}$ for $M=$ $2,3,5,6$, and 12 , respectively. For $M=3,5$, and 6 , the data points fall on a single curve for each $M$ to good approximation, while $M=2$ and 12 show some scattering. We can understand the ansatz (3) and the results in Fig. B in light of the qualitative arguments in Sec. IIIB We interpret $F_{\text {inf }}$ as the stall force in the stiff limit $k \rightarrow \infty$ and $U_{M}$ as the effect of thermal fluctuations, which induce slipping events and thereby lower the maximal force that the binding potential can support. The stiff limit $k \rightarrow \infty$ can be simulated by locking the motor subunits to their equilibrium positions. The results are shown in Fig. 3(b), using the above values for $U_{M}$. The stall force per bound motor subunit is consistent with Eq. (3) and $\lim _{x \rightarrow \infty} f_{M}(x)=1$ for $M=2,3,5$, and 6 . The case $M=12$ also gives a straight line for the stall force as a function of $U$ in the stiff limit, but neither consistent with $\lim _{x \rightarrow \infty} f_{12}(x)=1$ nor with the value of $U_{12}$ from finite stiffness. This illustrates the effect of dense motor subunits $(M>1 / a)$ in a stiff system.

The function $f_{M}\left(k d^{2} / U\right)$ is a normalized stall force per motor subunit. As is evident from Fig. 31(a), the force production per motor subunit varies strongly with stiffness and also with the number of subunits, $M$. Clearly, the interaction between the motor subunits is important for the force generation. The stall force is more complicated than a sum of contributions from the individual parts. According to the arguments in Sec. IIIB one expects $f_{M}(x)$ to be an increasing function of $x$ for $M<1 / a$, since larger stiffness decreases the probability that the unbound motor subunit binds to the wrong
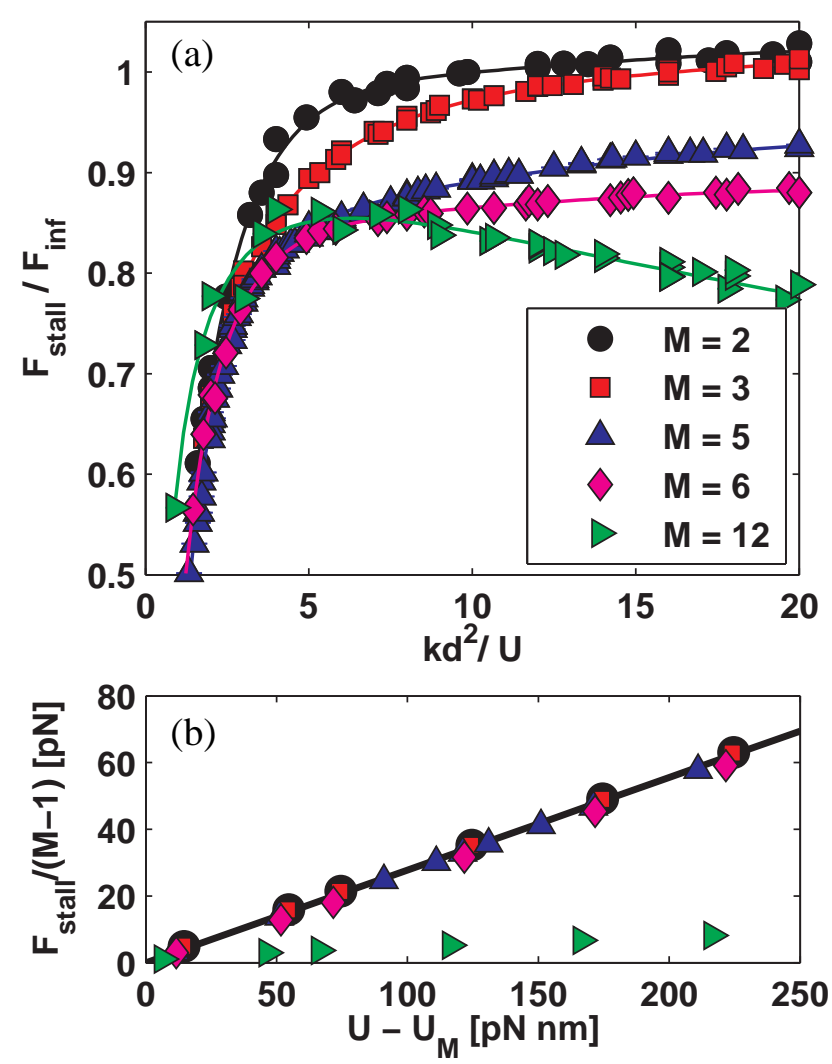

Figure 3: (Color online) (a) Stall force as a function of $k / U$ in the range $5<k<250 \mathrm{pN} / \mathrm{nm}$ and $80<U<200 \mathrm{pN} \mathrm{nm}$. The normalization $F_{\text {inf }}$ is chosen according to Eq. (3). Lines are guides to the eye. The case of many subunits $(M=12)$ is qualitatively different from the other cases. Here, the stall force depends nonmonotonically on $k / U$. (b) Stall force per bound motor subunit in the stiff limit $k \rightarrow \infty$ as a function of $U-U_{M}$. For $M \leq 6$ the simulations are consistent with $f_{M}(\infty)=1$ in Eq. (3) (solid line). $M=12$ gives a lower stall force that does not fit Eq. (3), which again illustrates the nonlinear behavior of the stall force on $M$. Error bars in both graphs are smaller than the symbols.

potential well. For $M>1 / a$, the stall force is expected to have a maximum as a function of stiffness, reflecting the inset of the qualitatively different behavior at high stiffness. These expectations are consistent with Fig. 3 where $f_{2}(x), f_{3}(x), f_{5}(x)$, and $f_{6}(x)$ are monotonically increasing in the simulated region, whereas $f_{12}(x)$ has a maximum around 1.5. To confirm that dense motor subunits give lower stall force also for lower values of $M$, we performed simulations with $a=0.3$ and found that with $U=200 \mathrm{pN} n \mathrm{~nm}$ and $M=5$, a maximum stall force occurs for stiffness between 40 and $150 \mathrm{pN} / \mathrm{nm}$ (not shown), as expected. 


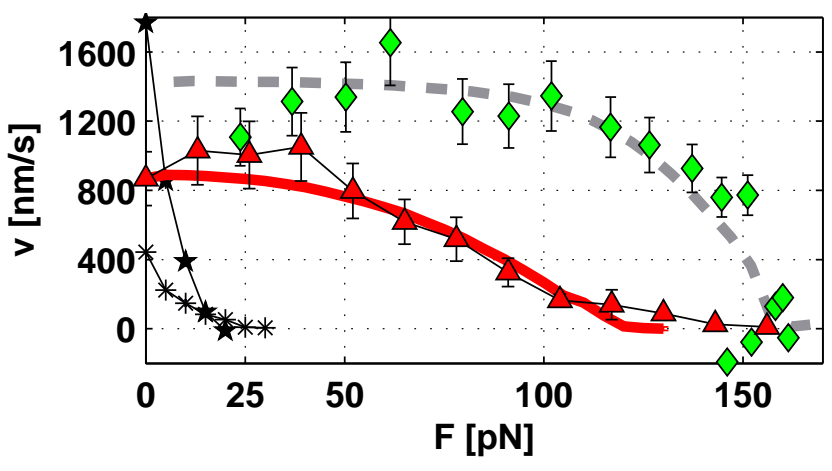

Figure 4: (Color online) Force-velocity characteristics from simulation and experiment. Triangles: average experimental velocity from Fig. 3(b) of Ref. [10]. Thick solid curve: $M=5$ model with $U=200 \mathrm{pN} \mathrm{nm}, k=25 \mathrm{pN} / \mathrm{nm}, F_{\text {stall }}=123 \mathrm{pN}$, and $\lambda=1180 \mathrm{~s}^{-1}$. Diamonds: a single retraction event from Ref. 8]. Dashed curve: $M=5$ model with $U=200 \mathrm{pN} \mathrm{nm}$, $k=60 \mathrm{pN} / \mathrm{nm}, F_{\text {stall }}=160 \mathrm{pN}$, and $\lambda=1800 \mathrm{~s}^{-1}$. Isolated motor subunits - i.e., simple flashing ratchet models (black symbols) are qualitatively different, as illustrated for $M=1$, $U=200 \mathrm{pN} \mathrm{nm}, \lambda=2000 \mathrm{~s}^{-1}$, and $t_{\text {off }}=25 \mathrm{~ms}(\star)$ or 2.5 $\mathrm{ms}(*)$.

\section{Comparison with an individual motor subunit}

To further highlight the effect of interactions and correlations, it is interesting to compare the interacting ring model to an isolated motor subunit. This is a single particle in a flashing ratchet potential and a special case of the interacting model, with $M=1$ and $k \rightarrow \infty$. This single-particle flashing ratchet (SPR) has been studied extensively [4, 39] in different versions, and its properties are qualitatively different from those of the interacting model in several interesting respects. For an SPR in this simple version, the mechanism to pull the particle to the next potential well is not present. Instead, forward motion relies on thermal noise to make the particle diffuse forward while in the unbound state. This means that the probability for a forward step can never exceed $1 / 2$ for a single chemical cycle even without applied force. With nonzero applied force, the free diffusion is superimposed on a backward motion with velocity $v_{\text {drift }}=-F / \gamma$; hence, the velocity is substantially reduced even at very low forces. These features conspire to make both the maximal velocity and the stall force depend strongly on the friction constant, the reaction rate, and how much time the particle spends unbound during a reaction cycle [13, 39]. Two examples of force-velocity relations for SPR are shown in Fig. 4 with $4 \mathrm{~nm}$ periodicity, $U=160$ $\mathrm{pN} n \mathrm{~m}, \lambda=2000 \mathrm{~s}^{-1}$, and damping as above. The transitions between the bound and unbound states are deterministic in these cases, and the time $t_{\text {off }}$ spent unbound during each cycle was $2.5 \mathrm{~ms}$ and $25 \mathrm{~ms}$.

\section{E. Application to a real motor system}

In Fig. 4 we compare model results with experimental data on $N$. gonorrhoeae. Several force-velocity relations averaged over many retraction events, as well as two single events, are preesented in Ref. [8, 10]. The maximum forces produced in the different events are distributed between 40 and $160 \mathrm{pN}$. There are several experimental factors that can make the maximum measured force in a particular retraction event lower than the intrinsic stall force $8,[10]$. These factors include, for example, breakage of the filament and are not included in the model. Accordingly, the model should be compared to the data that reach the highest forces. This leaves one single trajectory [8], shown in Fig. 4] and several average curves. The average force-velocity data in Fig. 4 represent an average of the data in Fig. 3 of Ref. [10]. Both experimental curves give positive velocities up to about $160 \mathrm{pN}$, which is in the upper tail of the maximum force distribution [10], but the velocity at lower forces differs considerably between the two curves.

To describe the PilT system, there are two natural choices for $M$ : namely, $M=5$, reflecting the five-fold symmetry of the filament, and $M=6$, reflecting the six-fold symmetry of the PilT molecule. We present simulation results for $M=5$, but $M=6$ is qualitatively no different.

The model can describe both experimental curves to some extent. The parameter values that describe the single event and the average data (see Fig. 4) differ in reaction $\lambda$ rate and stiffness $k$. There are two important deviations, which we discuss next.

The average velocity falls off exponentially at high forces [10], while the results of the model decay faster. The exponential decay can be described by an Arrhenius law for the rate limiting step 10]. The present model does not account for this behavior.

The single retraction event has a different decay at high forces and agrees better with the characteristics of the model. However, the single event shows an initial increase in velocity at low forces, rather than a plateau. Such initial increases in velocity are also obtained in some individual simulation runs, but disappear when the average is computed. All the experimental data also suffer from a possible systematic underestimation of the velocity near $F=0[8,10]$. Within the experimental accuracy, the general trend is a constant velocity up to about $50 \mathrm{pN}$ [8, 10], which is consistent with the results of the model.

\section{F. Deformations and elastic approximation}

From the results presented in Figs. 3 and 4 and Eq. (3), it is possible to estimate the magnitude of the deformations $y_{i}$ of the motor complex near the stall force. This is useful, since the harmonic approximation for the confining force on the motor subunits is questionable for large displacements. At the stall force, with $M-1$ sub- 
units bound to the filament, the mean displacement of a subunit can be estimated to $F_{\text {stall }} / k(M-1)$. As seen earlier, the stall force is less than $(M-1) U / d(1-a)$, which gives a displacement less than $U / k d(1-a)$. From Fig. 3 the interesting and relevant regime with high normalized stall force has $k d / U \gtrsim 1 \mathrm{~nm}^{-1}$. This gives a displacement less than $1 \mathrm{~nm}$ near the stall force. This is not excessively large compared to the dimensions of the PilT ring, whose diameter is $11.5 \mathrm{~nm}[33]$.

\section{G. Role of order in the binding process}

We next examine the effect of the ad hoc assumption of a sequential binding process on the results of the model. We compared the ordered sequence with two less correlated binding schemes. For random order with only one subunit free at the same time, the stall force is essentially unchanged, but the velocity decreases with about $50 \%$. Alternatively, if the binding and unbinding events are assumed to be independent for the different subunits, all subunits might occasionally become unbound simultaneously, which releases the filament from the motor. The resulting retraction events become highly irregular and have low stall force and mean velocity. We conclude that some degree of correlation between the motor subunits is necessary in order for the model to simulate the experiments.

\section{DISCUSSION AND CONCLUSION}

We investigate a model for force generation in finite ensembles of motor subunits interacting through an elastic backbone, which is inspired by the pilus retraction machinery, the strongest molecular motor reported so far. The model is prototypical, rather than realistic in detail, and offers a possible mechanism for generation of large retraction forces. It includes a ring of motor subunits surrounding the pilus filament, following a suggestion in Ref. 35]. Some parameters in the model can be roughly estimated, and we explore parts of the remaining parameter space and focus on generation of large forces.

We find that the stall force depends on the binding strength $U$ between motor and filament, the stiffness $k$ of the motor complex, the number of motor subunits $M$, and an asymmetry parameter $a$. For high enough stiffness we find qualitatively different properties compared to the well-studied model of a single particle in a flashing ratchet potential, which is the basic building block of our model. This is not surprising, since the mechanisms that generate motion are different in the two cases. The motion in the flashing ratchet model is dependent on diffusion [4]. Our model also contains diffusive motion of the motor subunits, but diffusion is not necessary for the motor to work [24, 25]. The dependence of the stall force on $U, k$, and $M$ is well parametrized in empirical scaling plots (Fig. 3). The scaling ansatz in Eq. (3) relies on the presence of an interaction between the motor subunits, and the stall force depends nonlinearly on the number of motor subunits.

Low stiffness compared to binding strength has a strong destructive effect on the force production. For a small number of motor subunits, the stall force increases monotonically with increasing stiffness, but when the motor subunits become dense enough there is a crossover to a different regime, where a high stiffness instead has a destructive effect on the stall force. For the asymmetry studied here, the crossover occurs around $M=1 / a$. For $M=5$ or 6 , corresponding to the number of filament strands or PilT subunits, a system with $a>0.2$ would have the interesting property that the maximum stall force is obtained for finite stiffness. If the binding strength between the PilT subunits can be genetically engineered, it might be possible to observe this effect in future experiments.

We compare results from the motor model for the filament retraction force-velocity characteristics with experiments on $N$. gonorrhoeae [8, 10]. Since the molecular details of the retraction mechanism are unknown, it is unclear to what extent the agreement we see reflects actual similarities between the model and the real system. More information would be useful for the construction of more detailed models. Nevertheless, the model can describe the general features of the experimental results i.e., the plateau in the force-velocity relation at low applied forces and the high stall force that is independent of reaction rate [8, 10].

For a quantitative comparison we select two different experimental force-velocity relations that show large forces, one single event, and one averaged curve, as shown

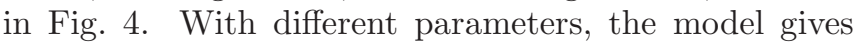
a reasonable description of the average data, as well as what looks like an atypical single event. The model deviates from the average data at high forces, which indicates that something is missing from the description. However, the data might include variations in cellular conditions that affect the average at high forces. This is not accounted for in the simulations, where the average is taken over thermal fluctuations with fixed parameters. The single event is described better and does not suffer from such a complication. In this case, the model is limited at high forces mainly by thermally assisted transitions of motor subunits between potential minima. Given the simplicity of the model, we find the agreement with experiments encouaging.

Small ensembles of interaction motor systems have previously been found to possess rich behavior without applied force 24, 25, 27]. As we have shown, this is true also in the limit of high forces, which is a realistic experimental situation. Generation of strong forces in nanoscale devices is also of technological interest, and it is tempting to speculate about the possibility to realize a setup of interacting motors units pulling on an artificial filament such as a carbon nano tube. 


\section{Acknowledgments}

The authors thank Berenike Maier for valuable discussions and comments. This work was supported by the Swedish Research Council [Grants Nos. 2003-5001
(M.W.) 2001-6456, 2002-6240, and 2004-4831 (A.B.J.)], the Royal Institute of Technology, the Göran Gustafsson Foundation, the Swedish Cancer Society, and Uppsala University.
[1] M. P. Sheetz, ed., Laser Tweezers in Cell Biology, vol. 55 of Methods in Cell Biology (Academic Press, San Diego CA, U.S.A., 1998).

[2] D. Boal, Mechanics of the Cell (Cambridge University Press, Cambridge, England, 2002), chap. 9.

[3] J. Howard, Mechanics of Motor Proteins and the Cytoskeleton (Sinauer Associates Inc., Sunderland MA, U.S.A., 2001).

[4] P. Reimann, Phys. Rep. 361, 57 (2002).

[5] C. Bustamante, D. Keller, and G. Oster, Acc. Chem. Res. 34, 412 (2001).

[6] M. Schliwa and G. Woehlke, Nature (London) 422, 759 (2003).

[7] J. S. Mattick, Annu. Rev. Microbiol. 56, 289 (2002).

[8] B. Maier, L. Potter, M. So, H. S. Seifert, and M. P. Sheetz, Proc. Natl. Acad. Sci. 99, 16012 (2002).

[9] A. J. Merz, M. So, and M. P. Sheetz, Nature (London) 407, 98 (2000).

[10] B. Maier, M. Koomey, and M. P. Sheetz, Proc. Natl. Acad. Sci. USA 101, 10961 (2004).

[11] F. Jülicher, A. Ajdari, and J. Prost, Rev. Mod. Phys. 69, 1269 (1997).

[12] G. Lattanzi and A. Maritan, Phys. Rev. Lett. 86, 001134 (2001).

[13] R. D. Astumian and M. Bier, Phys. Rev. Lett. 72, 1766 (1994).

[14] Y. Shu and H. Shi, Phys. Rev. E 69, 021912 (2004).

[15] M. Badoual, F. Jülicher, and J. Prost, Proc. Natl. Acad. Sci. 99, 6696 (2002).

[16] R. Lipowsky, S. Klumpp, and T. M. Nieuwenhuizen, Phys. Rev. Lett. 87, 108101 (2001).

[17] F. Jülicher and J. Prost, Phys. Rev. Lett. 75, 2618 (1995).

[18] F. Jülicher and J. Prost, Phys. Rev. Lett. 78, 4510 (1997).

[19] Y. Aghababaie, G. I. Menon, and M. Plischke, Phys. Rev. E 59, 2578 (1999).

[20] F. Jülicher, A. Ajdari, and J. Prost, Rev. Mod. Phys. 69, 1269 (1997).
[21] I. Derényi and T. Vicsek, Proc. Natl. Acad. Sci. 93, 6775 (1996).

[22] A. Vilfan, E. Frey, and F. Schwabl, Europhys. Lett. 45, 283 (1999).

[23] Z. Csahók, F. Family, and T. Vicsek, Phys. Rev. E 55, 5179 (1997).

[24] D. Dan, A. M. Jayannavar, and G. I. Menon, Physica A 318, 40 (2003).

[25] S. Klumpp, A. Mielke, and C. Wald, Phys. Rev. E 63, 031914 (2001).

[26] R. B. J. Xing, F. Bai and G. Oster, Proc. Natl. Acad. Sci. 103, 1260 (2006).

[27] E. B. Stukalin, H. Phillips III, and A. B. Kolomeisky, Phys. Rev. Lett. 94, 238101 (2005).

[28] K. T. Forest and J. A. Tainer, Gene 192, 165 (1997).

[29] H. Sun, D. R. Zusman, and W. Shi, Curr. Biol. 10, 1143 (2000).

[30] J. M. Skerker and H. C. Berg, Proc. Natl. Acad. Sci. USA 98, 6901 (2001).

[31] M. Dogterom, M. Janson, C. Faivre-Moskalenko, A. van der Horst, J. Kerssemakers, C. Tanase, and B. Mulder, Appl. Phys. A: 75, 331 (2002).

[32] M. E. Janson and M. Dogterom, Phys. Rev. Lett. 92, 248101 (2004).

[33] K. T. Forest, K. A. Satyshur, G. A. Worzalla, J. K. Hansen, and T. J. Herdendorf, Acta Crystallogr., Sect. D 60, 978 (2004).

[34] N. LeBrasseur, J. Cell Biol. 159, 912a (2002).

[35] D. Kaiser, Curr. Biol. 10, R777 (2000).

[36] H. E. Parge, K. T. Forest, M. J. Hickey, D. A. Christensen, E. D. Getzoff, and J. A. Tainer, Nature (London) 378, 32 (1995).

[37] P. E. Kloeden and E. Platen, Numerical Solution of Stochastic Differential Equations (Springer Verlag, Berlin, 1992).

[38] J. M. R. Parrondo, J. M. Blanco, F. J. Cao, and R. Brito, Europhys. Lett. 43, 248 (1998).

[39] R. D. Astumian, Science 276, 917 (1997). 